\title{
A contribuição do Brasil para a pesquisa eḿ atividade física e saúde no cenário internacional
}

\section{Brazilian contribution to research on physical activity and health in the international scenario}

\author{
Bruna Gonçalves Cordeiro da Silva ${ }^{1}$ \\ Juan Pablo Rey-López² \\ Fernando Pires Hartwig ${ }^{1}$ \\ Thiago Gonzalez Barbosa-Silva' \\ Renata Moraes Bielemann \\ Carolina de Vargas Nunes Coll ${ }^{1}$
}

\section{RESUMO}

O objetivo do presente estudo foi verificar a contribuição da produção científica brasileira na área de atividade física e saúde em periódicos internacionais. Foi realizada uma busca por artigos publicados na área de atividade física e saúde no período de 2009 a 2013 em 10 periódicos de relevância para a área. As publicações brasileiras na área foram contabilizadas e descritas por periódico, ano, eixo de pesquisa e localidade de afiliação dos autores. Foram encontradas 55 publicações brasileiras (2,4\%) no período analisado. Os principais eixos de pesquisa contemplados nessas publicações foram "correlatos à prática de atividade física" (44,8\%) e "níveis de atividade física e tendências temporais" (29,3\%). Além disso, as publicações estiveram concentradas em três cidades das regiões Sul e Sudeste. Foi evidenciada estável, porém importante, participação brasileira nas publicações internacionais na área de atividade física e saúde nos últimos cinco anos, com concentração em poucos centros de pesquisa e estudos pouco diversificados. Para aumentar a inserção internacional brasileira na área de atividade física e saúde, aponta-se a necessidade de diversificação dos temas de pesquisa e a descentralização da produção científica, visando gerar evidências extrapoláveis para outras populações e ampliar a capacidade nacional de conduzir pesquisas relevantes e de interesse internacional.

\section{PALAVRAS-CHAVE}

Atividade motora; Saúde; Publicações; Pesquisa; Epidemiologia; Brasil.

\begin{abstract}
The aim of the present study was to evaluate the Brazilian scientific production contribution to the field of physical activity and health in the international scenario. A search was performed for published manuscripts in the field of physical activity and bealth in 10 scientific journals of relevance in the area, from 2009 to 2013. Brazilian publications were counted and described according to journal, year, research subarea and author's affiliations. 55 Brazilian publications $(2.4 \%$ of the total) were found for the considered time period. The main research subareas covered in these publications were "correlates of physical activity" (44.8\%) and "physical activity levels and time trends" (29.3\%). Moreover, such publications were concentrated in three cities on the South and Southeast regions. In conclusion, a stable but important Brazilian participation in international publications in the field of physical activity and health in the last five years was evidenced, with studies concentrated in a few research centers and poorly diversified. To increase the international insertion of Brazil in the field of physical activity and health, research diversification and decentralization are necessary, in order to generate evidence with external validity and to expand the national capacity to conduct studies of relevance and international interest.
\end{abstract}

\section{KEYWORDS}

Motor activity; Health; Publications; Research; Epidemiology; Brazil. 


\section{INTRODUCCÃO}

A inatividade física é reconhecida atualmente como um dos principais fatores de risco para o desenvolvimento de doenças crônicas não transmissíveis (DCNTs) ${ }^{1}$, sendo responsável por aproximadamente 5,3 milhões de mortes ao ano no mundo ${ }^{2}$. O debate existente hoje sobre a relação entre atividade física e promoção da saúde é resultante de um processo histórico no qual comportamentos relacionados a um estilo de vida saudável passaram a ser incluídos nas agendas de saúde pública dos países ${ }^{3,4}$.

O estudo da relação entre atividade física e saúde teve como marco inicial a publicação de Morris ${ }^{5}$. Após isso, observou-se importante transição na pesquisa em atividade física, quando o foco migrou da aptidão física voltada ao desempenho para a aptidão física relacionada à saúde em meados da década de $80^{6}$. Essa mudança não apenas modificou o olhar sobre a relação entre atividade física e saúde, mas também ampliou os interesses e necessidades de pesquisa acerca do tema.

No Brasil, o aumento no número de publicações em atividade física e saúde coincidiu com a participação de profissionais de Educação Física nos programas de pós-graduação de Saúde Coletiva e a inserção da Educação Física enquanto campo profissional da área da saúde em $2000^{6,7}$. Após estes acontecimentos, além do maior número de artigos, foram observados também um maior número de profissionais com tal formação, grupos de pesquisa e dissertações relacionadas à área ${ }^{6}$.

Embora o estudo da relação entre atividade física e saúde tenha demonstrado crescimento no país, pouco se sabe, quantitativamente, sobre a contribuição nacional nas publicações da área em periódicos internacionais. Dessa forma, o objetivo do presente estudo foi verificar a contribuição da produção científica brasileira na área de atividade física e saúde em periódicos internacionais, identificando também as temáticas investigadas e a distribuição geográfica dos pesquisadores responsáveis pelas publicações.

\section{MÉTODOS}

Para a análise proposta, 10 periódicos internacionais relevantes para a área de atividade física e saúde foram escolhidos por pesquisadores com destaque na produção científica relacionada (Quadro 1). Essa estratégia foi elaborada com base na metodologia utilizada previamente por Hallal e colaboradores ${ }^{8}$ para um propósito similar, porém voltado à América Latina, visando manter a comparabilidade entre os dois estudos.

QUADRO 1 - Periódicos selecionados para a busca de publicações.

- American Journal of Epidemiology (Am J Epidemiol)

- American Journal of Preventive Medicine (Am J Prev Med)

- American Journal of Public Health (Am J Public Health)

- British Journal of Sports Medicine (Br J Sports Med)

- International Journal of Behavioral Nutrition and Physical Activity (Int J Behav Nutr Phys Act)

- International Journal of Epidemiology (Int J Epidemiol)

- Journal of Epidemiology and Community Health (J Epidemiol Community Health)

- Journal of Physical Activity and Health (J Phys Act Health)

- Medicine \& Science in Sports \& Exercise (Med Sci Sports Exerc)

- Preventive Medicine (Prev Med) 
A busca foi realizada diretamente no website dos periódicos, onde foram examinados todos os números publicados nos últimos cinco anos (janeiro de 2009 a dezembro de 2013). No presente artigo, enquadraram-se como publicações na área de atividade física e saúde os artigos cujos objetivos envolviam a prática de atividade física como exposição ou desfecho primário, seus correlatos ou influência sobre desfechos relacionados à saúde (doenças, comportamentos, qualidade de vida, fatores de risco para doenças crônicas não transmissíveis). Todos os artigos originais ou de revisão na área de atividade física e saúde foram contabilizados; e, dentre esses, foram identificadas as publicações brasileiras. Foram consideradas publicações brasileiras aquelas que continham pelo menos um autor com afiliação brasileira ou cuja população estudada tenha sido do Brasil.

Posteriormente, seguindo os cinco eixos principais da área de atividade física e saúde apresentados por Florindo e Hallal em livro recente ${ }^{9}$, as publicações brasileiras encontradas foram classificadas em: 1) níveis de atividade física e tendências temporais; 2) correlatos à prática de atividade física; 3) consequências da prática de atividade física; 4) intervenções na área de atividade física e 5) plano de ação nacional e internacional. A definição do eixo de pesquisa de cada publicação foi definida com base no título da mesma e, em caso de dúvida, foi verificado também o seu resumo. Artigos que contemplaram mais de um eixo de pesquisa foram contabilizados uma vez em cada eixo.

Foi realizada uma análise descritiva dos dados com valores absolutos e relativos das publicações encontradas nos periódicos supracitados. Posteriormente, procedeu-se com a análise estratificada por ano, visando descrever a evolução temporal da participação brasileira no âmbito da literatura internacional sobre o tema, aplicando-se também um teste estatístico formal para avaliação de tendência linear. Contabilizaram-se, ainda, para cada artigo selecionado, as cidades dos centros de pesquisa aos quais os autores eram afiliados. A contagem foi feita através da soma do número de artigos em que cada cidade encontrada apareceu ao menos uma vez nas afiliações.

\section{RESULTADOS}

Os resultados da busca para cada periódico, no período de 2009 a 2013, são mostrados na Tabela 1 . No total, foram encontradas 2315 publicações na área de atividade física e saúde, das quais $55(2,4 \%)$ tiveram ao menos um autor afiliado a uma instituição brasileira ou estudaram essa população. A mediana da proporção de artigos brasileiros foi 2,4 (intervalo interquartil: 1,8-4,7). A menor proporção de publicações foi observada no periódico British fournal of Sports Medicine (0,7\%) e a maior, no American fournal of Public Health (7,7\%).

$\mathrm{Na}$ Figura 1 é mostrada a variação da frequência de artigos brasileiros por ano. A representatividade anual de publicações brasileiras na área de atividade física e saúde foi de 12 (2,6\%), 8 (1,9\%), 17 (3,5\%), 5 (1,0\%) e 13 (2,9\%) para os anos de 2009 a 2013, respectivamente, período em que não se observou qualquer tendência linear $(\mathrm{p}=0,849)$. A Figura 2 ilustra a representatividade de cada eixo de pesquisa nas publicações internacionais na área de atividade física e saúde com participação brasileira. O principal eixo foi o de "correlatos à prática de atividade física”, o qual foi contemplado 26 vezes $(44,8 \%)$, seguido por estudos de "níveis de atividade física e tendências temporais" ( $\mathrm{n}=17$; 
29,3\%). O eixo com menor representatividade foi o "plano de ação nacional e internacional”, contemplado em apenas uma publicação (1,7\%).

TABELA 1 - Publicações na área de atividade física e saúde, por periódico avaliado (2009-2013).

\begin{tabular}{lccc}
\hline \multicolumn{1}{c}{ Periódico } & Total (N) & Artigos brasileiros \\
\hline & & 1 & $\%$ \\
\hline Br J Sports Med & 137 & 5 & 0,7 \\
\hline Am J Prev Med & 404 & 8 & 1,2 \\
\hline Int J Behav Nutr Phys Act & 476 & 6 & 1,7 \\
\hline Med Sci Sports Exerc & 306 & 1 & 2,0 \\
\hline Am J Epidemiol & 48 & 8 & 2,1 \\
\hline Prev Med & 307 & 21 & 2,6 \\
\hline J Phys Act Health & 560 & 1 & 3,8 \\
\hline J Epidemiol Community Health & 20 & 1 & 5,0 \\
\hline Int J Epidemiol & 18 & 3 & 5,6 \\
\hline Am J Public Health & 39 & 55 & 7,7 \\
\hline Total & 2315 & & 2,4 \\
\hline
\end{tabular}

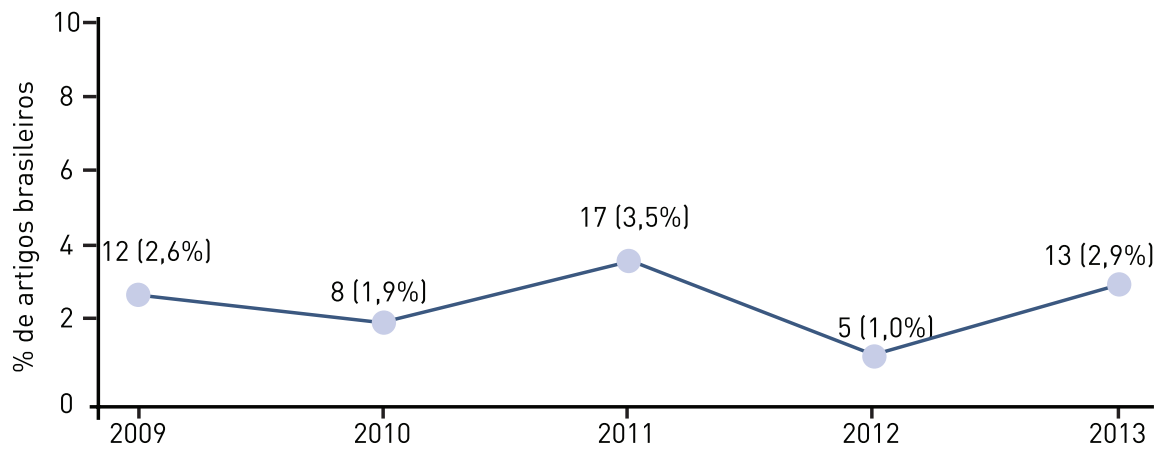

FIGURA 1 - Número de publicações brasileiras na área de atividade física e saúde nos periódicos selecionados, por ano.

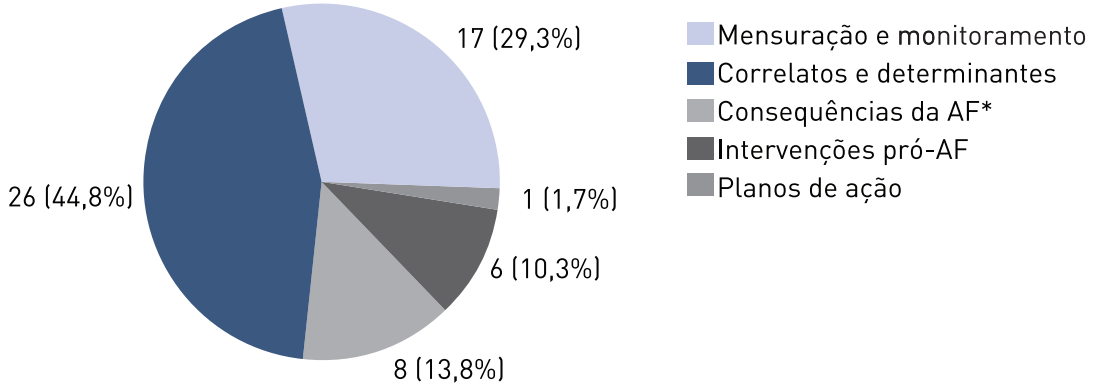

* Atividade Física

FIGURA 2 - Número de publicações brasileiras na área de atividade física e saúde nos periódicos selecionados (2009-2013), de acordo com o eixo de pesquisa.

A análise das cidades onde se localizam os centros de pesquisa envolvidos nas publicações dos artigos resultou em apenas 15 cidades diferentes (Figura 3). Duas publicações cujas coletas de dados ocorreram no Brasil, sem partici- 
pação de brasileiros na autoria, não foram incluídas nesta análise. Do total de 80 menções a cidades brasileiras nas afiliações dos autores dos artigos encontrados (independentemente de ser uma ou mais vezes por artigo), a maioria destas se concentrou nas cidades de Pelotas ( $\mathrm{n}=27 ; 33,6 \%)$, São Paulo ( $\mathrm{n}=14$; $17,5 \%)$ e Curitiba $(\mathrm{n}=11 ; 13,8 \%)$.

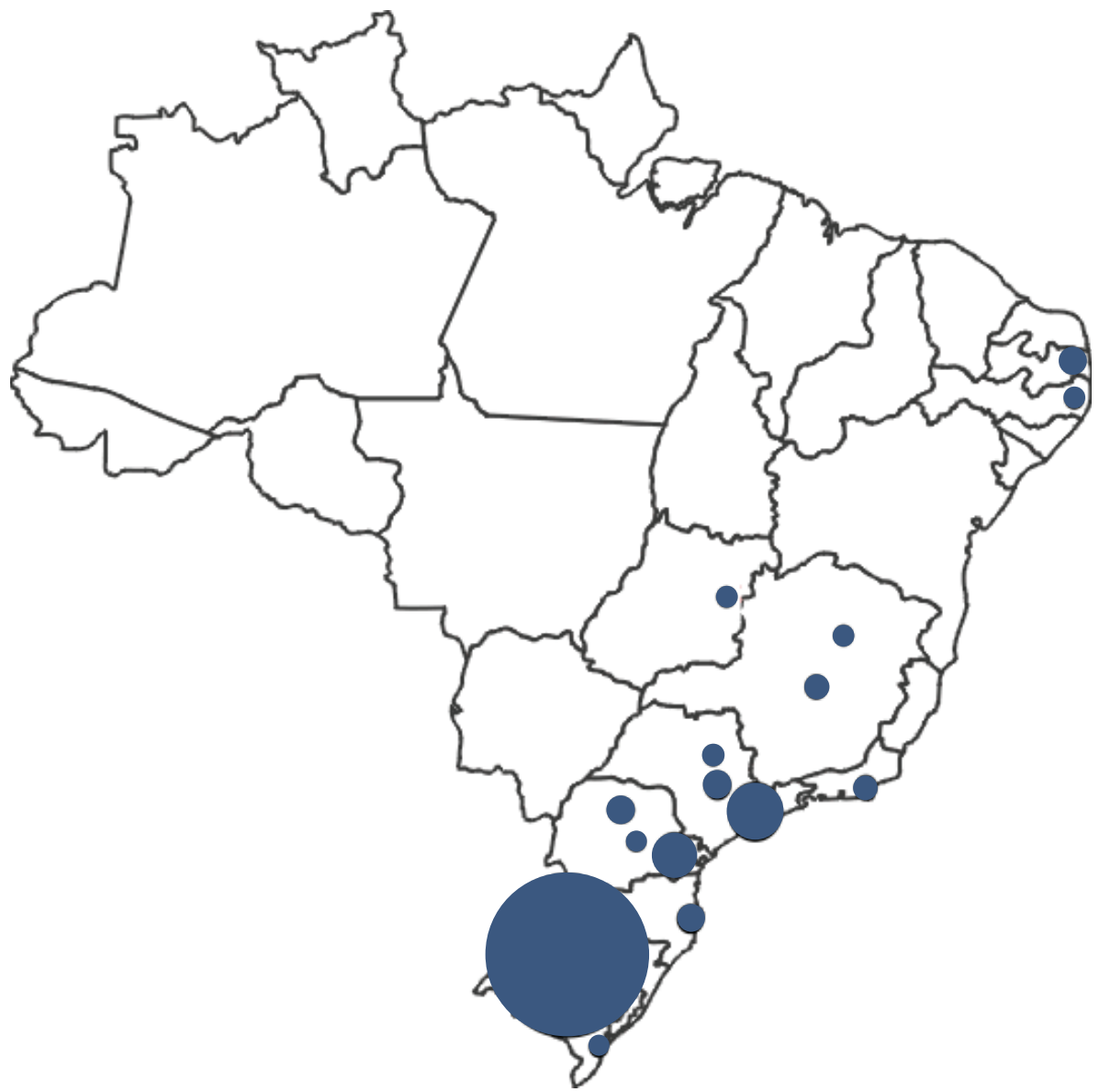

FIGURA 3 - Distribuição geográfica das publicações de acordo com as afiliações dos autores.

\section{DISCUSSÃO}

O presente estudo investigou a contribuição brasileira pelo número de publicações na área de atividade física e saúde em periódicos internacionais relevantes para a pesquisa na área. No período de investigação, não foi observada nenhuma tendência de incremento ou decréscimo na participação brasileira nesse tipo de publicação. Ainda, foi marcante o interesse brasileiro em estudos na temática de correlatos à prática de atividade física e evidenciou-se grande disparidade geográfica quanto à origem dos pesquisadores responsáveis pelas publicações, basicamente concentrados nas regiões Sul e Sudeste do país.

A criação da Sociedade Brasileira de Atividade Física e Saúde (SBAFS), que vem se fortalecendo nos últimos anos por meio da captação de membros e congressos bem estabelecidos, foi um marco importante para a consolidação da área no país ${ }^{10}$. No entanto, com base nos achados apresentados, observou-se que a participação do Brasil no cenário internacional de publicações 
científicas na área de atividade física e saúde ainda parece pequena quando observamos o total de publicações na área, no período estudado. Por outro lado, especulando que que a representação do Brasil na pesquisa mundial seja em torno de $0,6 \%$, considerando o total de artigos publicados na base de dados PubMed e a busca por afiliações com a palavra "Brazil" (busca realizada em 25 de abril de 2014), parece que a área de pesquisa em atividade física e saúde feita no Brasil tem maior visibilidade internacional quando comparada à outras áreas de pesquisa.

O estudo de Hallal e colaboradores ${ }^{8}$, anteriormente, evidenciou uma baixa produção científica da América Latina (2\%) na literatura em atividade física e saúde mundial entre os anos de 2004 e 2009. No presente estudo encontramos que, nos cinco anos subsequentes, a participação brasileira no total de artigos na área de atividade física e saúde foi um pouco superior a essa (2,4\%). Esse resultado pode indicar um aumento na participação latino-americana em todo o escopo de publicações da área ou, ainda, a visibilidade do Brasil na área em relação aos demais países que compõem a América Latina.

No contexto internacional, dois bons exemplos do aumento da relevância brasileira na área foram a realização do Congresso Internacional de Atividade Física e Saúde Pública no Rio de Janeiro (Brasil) em 2014 e a significativa participação de pesquisadores brasileiros no suplemento especial de atividade física do The Lancet, um dos periódicos científicos mais bem conceituados na área da saúde, em 2012 $2^{1,11-13}$. Devido ao crescimento da área de atividade física e saúde no país ${ }^{6}$, somado ao aumento da sua visibilidade no mundo, era esperado que se encontrasse um aumento nas publicações brasileiras da área no cenário internacional nos últimos anos. No entanto, a contribuição do Brasil no total de publicações na área, conforme avaliada no presente estudo, variou pouco entre os anos de 2009 e 2013.

De fato, o referido crescimento parece não ter sido suficiente para favorecer uma maior inserção da pesquisa brasileira em periódicos internacionais, ao menos nos últimos cinco anos. Alguns motivos que podem ter influenciado esse resultado são a dificuldade de alguns pesquisadores brasileiros com a redação em inglês, a especificidade de alguns resultados para a realidade local ${ }^{14}$ e o fato da maioria dos artigos brasileiros da área de atividade física e saúde possuírem delineamento transversal, os quais são muitas vezes menos valorizados na literatura internacional do que estudos com delineamentos experimentais ${ }^{15}$. Ademais, a distância geográfica ${ }^{16}$ pode também ser um fator que dificulta o crescimento das publicações brasileiras da área, uma vez que o Brasil está geograficamente distante de grandes centros de referência em pesquisa, como Estados Unidos e Inglaterra. Da mesma forma, a região norte do Brasil localiza-se consideravelmente distante das cidades detectadas com maior número de autores das publicações encontradas, o que assim contribui também para a iniquidade regional observada, provavelmente comum a outras áreas de pesquisa.

Os principais eixos de pesquisa das publicações brasileiras nos periódicos internacionais foram "correlatos" e "níveis e tendências temporais", o que já foi mostrado em editorial recente da Revista Brasileira de Atividade Física e Saúde ${ }^{17}$. Tal achado pode ser meramente um reflexo da produção de conhecimento da área no país, que apesar de ter evoluído muito nos últimos anos, 
ainda não tem uma produção científica expressiva nos demais eixos de pesquisa, conforme observado em estudos epidemiológicos direcionados ao estudo da atividade física ${ }^{18}$. Ressalta-se também que as pesquisas desenvolvidas nos eixos de "consequências", "intervenções" e "ações" geralmente exigem delineamentos de estudo mais robustos e/ou a captação de grandes recursos financeiros. Além disso, cabe salientar que era pouco esperado que se encontrasse expressiva publicação internacional no eixo de pesquisa "ações", uma vez que normalmente essas são mais encontradas em relatórios técnicos e documentos governamentais de maior alcance regional.

A necessidade de descentralização das pesquisas na área de atividade física e saúde é outro fato que chama atenção, visto que a maioria dessas está concentrada em apenas três cidades, as quais contemplam apenas as regiões Sul e Sudeste do país. Estas disparidades regionais podem ser causa ou consequência da já observada iniquidade no Brasil na instalação de Programas de Pós-Graduação, distribuição de recursos financeiros e grupos de pesquisa, não somente da área de atividade física e saúde ${ }^{19,20}$, mas também da educação física em geral $^{21}$.

Como limitações do estudo, cabe salientar que a opinião dos pesquisadores consultados em relação à escolha dos periódicos a serem incluídos na busca pode ter sido influenciada por aspectos subjetivos, tais como suas preferências para publicação. Entretanto, destaca-se a possibilidade de comparação com o editorial anterior ${ }^{8} \mathrm{e}$ o fato de que os periódicos selecionados, além de possuírem bons fatores de impacto (de 1,95 a 6,98), corroborando a importância internacional da escolha dos mesmos, também são periódicos de grande relevância à literatura mundial no campo da atividade e saúde. Ainda, em uma análise na base de dados PubMed, foi visto que do total de artigos encontrados com o termo "physical activity" entre $1^{\circ}$ de janeiro de 2009 e 31 de dezembro de 2013, 2,6\% são ainda localizados junto da palavra "Brazil", percentual similar àquele encontrado no presente estudo (2,4\%) (busca realizada em 04 de maio de 2014).

Sendo assim, foi evidenciada estável, porém importante, participação brasileira nas publicações internacionais na área de atividade física e saúde nos últimos cinco anos. Foi observada uma concentração dos pesquisadores da área em poucos centros de pesquisa do país e também maior número de publicações sobre correlatos à prática de atividade física e níveis de atividade física e tendências temporais. Para aumentar a inserção e para que não haja um decréscimo da participação internacional brasileira na área de atividade física e saúde nos próximos anos, aponta-se a necessidade de diversificação dos eixos de pesquisa, principalmente a realização de mais estudos longitudinais e experimentais; a descentralização da produção científica, através da capacitação de recursos humanos em instituições incipientes na área e ampliação de redes de colaboração com pesquisadores e centros de pesquisas internacionais. Com isso, também será possível gerar mais evidências extrapoláveis para outras populações e ampliar a capacidade nacional de conduzir pesquisas relevantes e de interesse internacional.

\section{Agradecimentos}

Os autores gostariam de agradecer a colaboração do Dr. Pedro Curi Hallal na orientação metodológica do estudo apresentado. Também ressaltam agra- 
decimento às seguintes instituições financiadoras de pesquisa pelo suporte financeiro através de bolsas de pós-graduação individuais para os envolvidos: CNPq, CAPES, FAPERGS e FAPESP.

\section{Contribuição dos autores}

RMB foi responsável pela definição do tema, redação e revisão do artigo. BGCS, JPRL, FPH, TGBS e CVNC foram responsáveis pela pesquisa nos periódicos, elaboração do texto e revisão do artigo. BGCS e CVNC foram responsáveis pela divisão dos artigos por áreas do conhecimento. JPRL foi responsável pela divisão dos artigos por localidade geográfica. FPH foi responsável pela análise estatística dos dados. BGCS, FPH e TGBS foram responsáveis pela elaboração das figuras.

\section{REFERÊNCIAS}

1. Hallal PC, Andersen LB, Bull FC, Guthold R, Haskell W, Ekelund U. Global physical activity levels: surveillance progress, pitfalls, and prospects. Lancet 2012;380(9838):247-257.

2. Lee IM, Shiroma EJ, Lobelo F, Puska P, Blair SN, Katzmarzyk PT. Effect of physical inactivity on major non-communicable diseases worldwide: an analysis of burden of disease and life expectancy. Lancet 2012;380(9838):219-229.

3. WORLD HEALTH ORGANIZATION (WHO). The Ottawa charter on health promotion. Ottawa: WHO; 1986.

4. U.S. DEPARTMENT OF HEALTH AND HUMAN SERVICES. Physical activity and health: a report of the surgeon general. Atlanta: HHS/CDC; 1996.

5. Morris JN, Heady JA, Raffle PA, Roberts CG, Parks JW. Coronary heart-disease and physical activity of work. Lancet 1953;265(6795):1053-1057; contd.

6. Nahas MV, Garcia LMT. Um pouco de história, desenvolvimentos recentes e perspectivas para a pesquisa em atividade física e saúde no Brasil Rev bras Educ Fís Esporte 2010;24(1):135-148.

7. Hallal PC, Dumith Sde C, Bastos JP, Reichert FF, Siqueira FV, Azevedo MR. Evolução da pesquisa epidemiológica em atividade física no Brasil: revisão sistemática. Rev Saude Publica 2007;41(3):453-460.

8. Hallal PC, Parra DC, Azevedo MR, Pratt M, Brownson RC. Pesquisa em Atividade Física e Saúde: Onde está a América Latina? J Phys Act Health 2010;7 Suppl 2:S129-130.

9. Florindo AA, Hallal PC. Epidemiologia da Atividade Física. São Paulo; 2011.

10. Reis RS. Reflexões sobre o IX Congresso Brasileiro de Atividade Física e Saúde. Revista Brasileira de Atividade Física e Saúde 2013;18(5):521-522.

11. Das P, Horton R. Rethinking our approach to physical activity. Lancet 2012;380(9838):189-190.

12. Malta DC, Barbosa da Silva J. Policies to promote physical activity in Brazil. Lancet 2012;380(9838):195-196.

13. Rimmer JH, Marques AC. Physical activity for people with disabilities. Lancet 2012;380(9838):193-195.

14. Victora CG, Moreira CB. Publicações científicas e as relações Norte-Sul: racismo editorial? Rev Saude Publica 2006;40 Spec no.:36-42.

15. Victora CG, Habicht JP, Bryce J. Evidence-based public health: moving beyond randomized trials. Am J Public Health 2004;94(3):400-405.

16. Pan RK, Kaski K, Fortunato S. World citation and collaboration networks: uncovering the role of geography in science. Sci Rep 2012;2:902.

17. Florindo AA, Rombaldi AJ, Barros MVG. Análise da Revista Brasileira de Atividade Física e Saúde em 2013: O copo está meio cheio ou meio vazio? Revista Brasileira de Atividade Física e Saúde 2013;18(6):2. 
18. Ramires VV, Becker LA, Sadovsky ADI, Zago AM, Bielemann RM, Guerra PH. Evolução da pesquisa epidemiológica em atividade física no Brasil: atualização de uma revisão sistemática.

19. Garcia LMT, Böhm AW, Bacil EDA, Cruz MF, Santo RCE. A inserção da subárea de Atividade Física e Saúde nos programas de pós-graduação em Educação Física no Brasil. Revista Brasileira de Atividade Física e Saúde 2014;19(2):8.

20. Silva ICM. Pesquisa em atividade física e saúde no Brasil: um recorte dos investimentos em projetos e bolsas de produtividade CNPq.

21. Del Duca GF, Garcia LMT, Silva KS, Nascimento JV. Grupos de pesquisa em cursos de Educação Física com pós-graduação “stricto sensu” no Brasil: análise temporal de 2000 a 2008. Revista Brasileira de Educação Física e Esporte 2011;25:607-617.

\section{ENDEREÇO PARA}

CORRESPONDÊNCIA

CAROLINA DE VARGAS NUNES COLL

Rua Marechal Deodoro, 1660 ( $3^{\circ}$ andar).

CEP: 96020220 . Pelotas, RS. Telefone:

(053) 8125-0899.

E-mail: carolinavncollagmail.com 